\title{
Worst Case Pricing of Rainbow Options
}

\author{
Discussion Paper No. 217 \\ ISSN 0949-9962 \\ JEL C63, G13 \\ MSC 35K20, 65M60
}

Version October 12, 2001

\author{
Jürgen Topper: Andersen \\ Financial and Commodity Risk Consulting (FCRC) \\ Mergenthalerallee 55 \\ 65760 Eschborn/Frankfurt \\ Germany \\ Juergen.Topper@Andersen.com \\ Department of Economics (Mathematical Economics) \\ University of Hannover \\ Königsworther Platz \\ 30167 Hannover \\ Germany
}

I would like to thank Matthias Heurich, Christian T. Hille, Marian Maas, Vijay Parmar, Granville Sewell, Paul Wilmott and Uwe Wystup with respect to the discussions leading to the present article. A short version of this article has been published in [17]. 


\begin{abstract}
Options on two underlyings are a common exotic product in the equity and FX derivatives market. The value of these kinds of options depends on the correlation of the two underlyings. We will present a model to compute a lower bound for the price of this option. The model, represented by a non-linear parabolic PDE, is implemented with finite elements in order to demonstrate the results with several derivatives from the European market.
\end{abstract}

\title{
Contents
}

2 A Pricing Model with Uncertain Correlation 4

2.1 A Worst-Case Pricing Equation . . . . . . . . . . . . . . . . 4

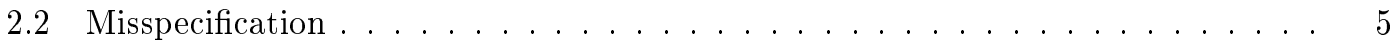

3 Implementing the Model

3.1 A Numerical Approach: The Collocation Finite Element Method . . . . . . . . 5

3.2 Estimating the Data . . . . . . . . . . . . . . . . . 7

4 Applications of the Model $\ldots$

4.1 Capped Calls on Baskets . . . . . . . . . . . . . . . . . . 8

4.2 Options on the Maximum or the Minimum of Two Risky Assets . . . . . . 9

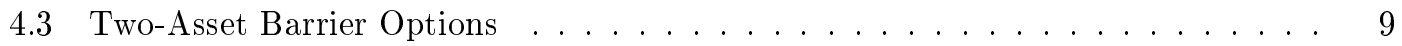

4.4 Basket Options . . . . . . . . . . . . . . . . . . . . . 10

5 Conclusions and Outlook $\quad 11$

$\begin{array}{ll}\text { Bibliography } & 12\end{array}$ 


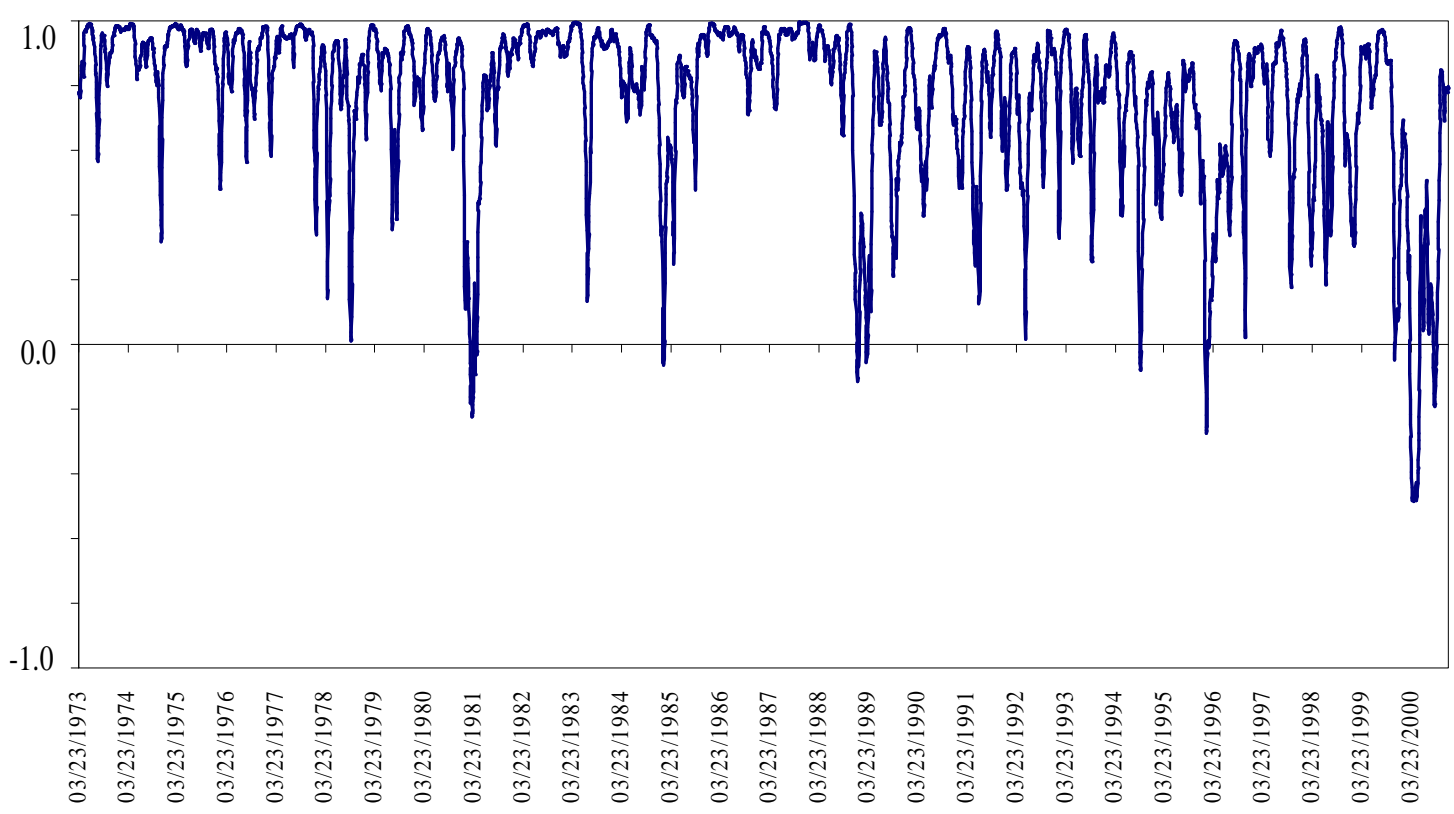

Figure 1: Historical 60-day Correlation between Commerzbank and Dresdner Bank

\section{Introduction}

Rainbow options on two assets or currencies are common in the derivatives market. In the OTC market plain vanilla basket options and digital basket options, sometimes with barriers or caps, are common exotics. Options on-the-worst-of-two are sometimes embedded in triple currency linked bonds or reverse convertibles. Triple linked currency bonds can be divided into a straight bond and a series of puts on the worst of two currencies [12]. So-called double reverse convertibles can be seen as a straight bond (sometimes a zero coupon bond) and a short position in a put on the worst of two equities. Products of this kind are currently popular in the retail market and are offered by BHF Bank and HSBC Trinkaus und Burkhardt. Reverse convertibles including a basket option on two equities as offered by $A B N$ Amro have not gained any popularity.

The price of an option on two assets depends strongly on the correlation of these two assets denoted by $\rho$. A straightforward extension of the classical Black-Scholes framework for options on two assets leads to the following well-known PDE [7]

$$
\begin{aligned}
\frac{1}{2} \sigma_{1}^{2} S_{1}^{2} \frac{\partial^{2} V}{\partial S_{1}^{2}}+\frac{1}{2} \sigma_{2}^{2} S_{2}^{2} \frac{\partial^{2} V}{\partial S_{2}^{2}} & +\rho \sigma_{1} \sigma_{2} S_{1} S_{2} \frac{\partial^{2} V}{\partial S_{1} \partial S_{2}}+ \\
\left(r-D_{1}\right) S_{1} \frac{\partial V}{\partial S_{1}}+\left(r-D_{2}\right) S_{2} \frac{\partial V}{\partial S_{2}} & =r V-\frac{\partial V}{\partial t}
\end{aligned}
$$

The problem, however, is that correlation $\rho$ is not a constant as can be seen in fig. 1. Instead of giving a correct figure for the correlation, it is much easier to derive a reasonable guess for the lower and upper bound. In the FX market, it is possible to compute implied correlation [9]. This, however, is usually not possible in the equity market.

In the following, we will show how to price an option when only upper and lower bounds for correlation are known. This approach uses similar hedging arguments as the original approach by Black and Scholes [1]. We are only interested in the worst-case solution. The derived pricing PDE is nonlinear in contrast to eq. (1). This model is similar in spirit to earlier models with respect to other unknown parameters such as volatility, dividends, and interest rates. For a survey see [19]. We will show how to implement this model using a collocation finite element method. This includes a thorough discussion of the appropriate boundary conditions. As examples, we present numerical results for several rainbow options from the European market. 


\section{A Pricing Model with Uncertain Correlation}

\subsection{A Worst-Case Pricing Equation}

Assuming that a lower and an upper bound for correlation is

$$
-1 \leq \rho^{-} \leq \rho \leq \rho^{+} \leq 1
$$

we shall use a similar argument as Black and Scholes [1] to derive a model for worst case pricing. Both assets follow the usual SDE:

$$
\begin{aligned}
& d S_{1}=\mu_{1} S_{1} d t+\sigma_{1} S_{1} d X \\
& d S_{2}=\mu_{2} S_{2} d t+\sigma_{2} S_{2} d X
\end{aligned}
$$

Both assets are correlated

$$
E\left(d X_{i} d X_{j}\right)=\rho d t
$$

Setting up a portfolio consisting of a long position in one option and short positions in both underlyings leads to:

$$
\Pi=V\left(S_{1}, S_{2}, t\right)-\Delta_{1} S_{1}-\Delta_{2} S_{2}
$$

With Itô's Lemma an infinitesimal change in this portfolio can be expressed as:

$$
d \Pi=\left(\frac{\partial V}{\partial t}+\frac{1}{2} \sum_{i=1}^{2} \sum_{j=1}^{2} \sigma_{i} \sigma_{j} \rho_{i j} S_{i} S_{j} \frac{\partial^{2} V}{\partial S_{i} \partial S_{j}}\right) d t+\sum_{i=1}^{2}\left(\frac{\partial V}{\partial S_{i}}-\Delta_{i}\right) d S_{i}
$$

This expression can be simplified because $i, j=2$. We also know that $\rho_{i i}=1$ and $\rho_{i j}=\rho_{j i}$. To simplify the notation we set $\rho_{12}=\rho_{21}=\rho$.

$$
\begin{aligned}
d \Pi & =\left(\frac{\partial V}{\partial t}+\sigma_{1} \sigma_{2} \rho S_{1} S_{2} \frac{\partial^{2} V}{\partial S_{1} \partial S_{2}}+\frac{1}{2} \sigma_{1}^{2} S_{1}^{2} \frac{\partial^{2} V}{\partial S_{1}^{2}}+\frac{1}{2} \sigma_{2}^{2} S_{2}^{2} \frac{\partial^{2} V}{\partial S_{2}^{2}}\right) d t \\
& +\left(\frac{\partial V}{\partial S_{1}}-\Delta_{1}\right) d S_{1}+\left(\frac{\partial V}{\partial S_{2}}-\Delta_{2}\right) d S_{2}
\end{aligned}
$$

Choosing $\Delta_{i}=\frac{\partial V}{\partial S_{i}}$ for $i=1,2$ elimininates the risk just as in the classical argument when deriving the multidimensional Black-Scholes equation.

$$
d \Pi=\left(\frac{\partial V}{\partial t}+\sigma_{1} \sigma_{2} \rho S_{1} S_{2} \frac{\partial^{2} V}{\partial S_{1} \partial S_{2}}+\frac{1}{2} \sigma_{1}^{2} S_{1}^{2} \frac{\partial^{2} V}{\partial S_{1}^{2}}+\frac{1}{2} \sigma_{2}^{2} S_{2}^{2} \frac{\partial^{2} V}{\partial S_{2}^{2}}\right) d t
$$

In the Black-Scholes model it can be argued that from knowing $V$ we also know $d \Pi$. Here, however, this is not true since $\rho$ is unknown. Since we want to derive a worst-case-scenario model, we want to be extremely pessimistic: In every infinitesimal time step we assume a correlation that leads to the smallest growth in the portfolio. This implies that the return on this worst-case portfolio is set equal to the risk-free rate.

$$
\min d \Pi \stackrel{!}{=} r \Pi d t
$$

The minimization is with respect to $\rho$. Using eq. (6) the right-hand side of eq. (10) can be rewritten as

$$
r \Pi d t=r\left(V\left(S_{1}, S_{2}, t\right)-\frac{\partial V}{\partial S_{1}} S_{1}-\frac{\partial V}{\partial S_{2}} S_{2}\right) d t
$$

The left-hand side can be expressed as

$$
\min _{\rho}\left[(\frac{\partial V}{\partial t}+\underbrace{\sigma_{1} \sigma_{2} \rho S_{1} S_{2} \frac{\partial^{2} V}{\partial S_{1} \partial S_{2}}}_{A}+\frac{1}{2} \sigma_{1}^{2} S_{1}^{2} \frac{\partial^{2} V}{\partial S_{1}^{2}}+\frac{1}{2} \sigma_{2}^{2} S_{2}^{2} \frac{\partial^{2} V}{\partial S_{2}^{2}}) d t\right]
$$

Because of the minimization with respect to $\rho \in\left[\rho^{-} ; \rho^{+}\right]$, we can distinguish two cases: 
1. $\frac{\partial V}{\partial S_{1} \partial S_{2}}=\Gamma_{\text {cross }}>0 \Rightarrow A=\rho^{-} \sigma_{1} \sigma_{2} S_{1} S_{2} \Gamma_{\text {cross }}$

2. $\frac{\partial V}{\partial S_{1} \partial S_{2}}=\Gamma_{\text {cross }}<0 \Rightarrow A=\rho^{+} \sigma_{1} \sigma_{2} S_{1} S_{2} \Gamma_{\text {cross }}$

Combining these results and taking dividends into account, we arrive at the worst-case pricing equation:

$$
\begin{aligned}
\frac{1}{2} \sigma_{1}^{2} S_{1}^{2} \frac{\partial^{2} V}{\partial S_{1}^{2}}+\frac{1}{2} \sigma_{2}^{2} S_{2}^{2} \frac{\partial^{2} V}{\partial S_{2}^{2}} & +\rho\left(\Gamma_{\text {cross }}\right) \sigma_{1} \sigma_{2} S_{1} S_{2} \frac{\partial^{2} V}{\partial S_{1} \partial S_{2}}+ \\
\left(r-D_{1}\right) S_{1} \frac{\partial V}{\partial S_{1}}+\left(r-D_{2}\right) S_{2} \frac{\partial V}{\partial S_{2}} & =r V-\frac{\partial V}{\partial t} \\
\rho\left(\Gamma_{\text {cross }}\right) & = \begin{cases}\rho^{-} & \text {if } \Gamma_{\text {cross }}>0 \\
\rho^{+} & \text {if } \Gamma_{\text {cross }}<0\end{cases} \\
\Gamma_{\text {cross }} & =\frac{\partial^{2} V}{\partial S_{1} \partial S_{2}}
\end{aligned}
$$

These results can be extended to $\rho^{-}$and $\rho^{+}$as they are deterministic functions of $t$. Obviously, it has to hold that $\rho^{-}(t)<\rho^{+}(t)$. It is also possible to combine this model with other models to adjust for the uncertainty of the other unknown parameters.

\subsection{Misspecification}

This section deals with the error incurred by estimating the bandwidth of $\rho$ in a too optimistic manner. We are interested in the effect of decreasing $\rho^{-}$and increasing $\rho^{+}$by infinitesimal amounts. The change in $V$ by relaxing $\rho^{-}$by $\epsilon$ is generally not the same as the change incurred by increasing $\rho^{+}$to $\rho^{+}+\epsilon$. Let us define:

$$
\begin{aligned}
\delta_{\rho^{-}}:=\frac{\partial V}{\partial \rho^{-}} & \approx \frac{V\left(\rho^{-}-\epsilon\right)-V\left(\rho^{-}+\epsilon\right)}{2 \epsilon} \\
\delta_{\rho^{+}}:=\frac{\partial V}{\partial \rho^{+}} & \approx \frac{V\left(\rho^{+}-\epsilon\right)-V\left(\rho^{+}+\epsilon\right)}{2 \epsilon} \\
\text { with } \epsilon & =0.01
\end{aligned}
$$

Both $\delta_{\rho^{-}}$and $\delta_{\rho^{+}}$are negative by definition: The price of an option in a worst-case scenario can only decrease by relaxing the input parameter.

\section{Implementing the Model}

\subsection{A Numerical Approach: The Collocation Finite Element Method}

These models are non-linear parabolic PDEs for which analytical solutions are not known. When, however, it can be shown that the Black-Scholes $\Gamma_{\text {cross }}$ is single-signed, the non-linear PDE reduces to the Black-Scholes pricing equation which is linear. For certain rainbow options it is well-known that $\Gamma_{\text {cross }}$ is single-signed so that only a linear PDE has to be solved. Sometimes, this can even be achieved analytically. From the large family of possible numerical solution techniques we have chosen a collocation finite element approach with cubic Hermite trial functions. This approximates $\Gamma_{\text {cross }}$ accurately and delivers the other hedging parameters as a by-product. From the finite difference family only explicit FD are straightforward to employ; ADI and hopscotch methods need to be extended ([19], ch. 48; [15]). For a possible extension of FDs to compute accurate cross-derivatives see [18].

First we consider the stationary problem $L(u(x, y))=f, L$ being a non-linear differential operator. We look for an approximate solution $\tilde{u}(x, y)$ for the following problem:

$$
\begin{aligned}
L(u) & =f \\
u\left(x_{\min }\right) & =u_{\min _{x}}, u\left(x_{\max }\right)=u_{\max _{x}}, \\
u\left(y_{\min }\right) & =u_{\min _{y}}, u\left(y_{\max }\right)=u_{\max _{y}}
\end{aligned}
$$


That is, we consider a rectangular domain with Dirichlet conditions. This method can easily be generalized for non-rectangular domains and Neumann and mixed boundary conditions [14]. An approximate solution $\tilde{u}$ can take the following form:

$$
\tilde{u}=\sum_{k=1}^{N} a_{k} \phi_{k}(x, y) \text {. }
$$

The task is to find values for $a_{i}$ which make $\tilde{u}$ the "best" approximation. While the Galerkin finite element methods sometimes used in two-asset derivative pricing (cf. [3], [16]) determine the $a_{i}$ 's by solving

$$
\int_{x_{\min }}^{x_{\max }} \int_{y_{\min }}^{y_{\max }}(L(\tilde{u})-f) \phi_{k} d y d x=0, k=1, \ldots, N,
$$

collocation finite element methods take the following approach. They enforce that, at certain points in the domain, the so-called collocation points, the PDE is exactly satisfied.

$$
(L(\tilde{u})-f)\left(\xi_{k}\right)=0, k=1, \ldots, N
$$

The domain is divided into rectangular disjoint elements, the finite elements. They are not necessarily of the same size. The four collocation points for each element $\left[x_{i} ; x_{i+1}\right] \times\left[y_{i} ; y_{i+1}\right]$ in the method used here are

$$
\left\{x_{i}+\left(\frac{1}{2} \pm \frac{1}{2 \sqrt{3}}\right)\left(x_{i+1}-x_{i}\right) ; y_{i}+\left(\frac{1}{2} \pm \frac{1}{2 \sqrt{3}}\right)\left(y_{i+1}-y_{i}\right)\right\}
$$

Let $s_{i}$ denote the number of gridlines in direction $i$. The approximate solution is defined by a linear combination of $4 s_{x} s_{y}$ basis functions

$$
H_{i}(x) H_{j}(y), H_{i}(x) S_{j}(y), S_{i}(x) H_{j}(y), S_{i}(x) S_{j}(y)
$$

with

$$
\begin{aligned}
& H_{k}(x)=3\left(\frac{x-x_{k-1}}{x_{k}-x_{k-1}}\right)^{2}-2\left(\frac{x-x_{k-1}}{x_{k}-x_{k-1}}\right)^{3} \text { for } x_{k-1} \leq x \leq x_{k} \\
& =3\left(\frac{x_{k+1}-x}{x_{k+1}-x_{k}}\right)^{2}-2\left(\frac{x_{k+1}-x}{x_{k+1}-x_{k}}\right)^{3} \text { for } x_{k} \leq x \leq x_{k+1} \\
& =0 \text { elsewhere } \\
& S_{k}(x)=-\frac{\left(x-x_{k-1}\right)^{2}}{\left(x_{k}-x_{k-1}\right)}+\frac{\left(x-x_{k-1}\right)^{3}}{\left(x_{k}-x_{k-1}\right)^{2}} \text { for } x_{k-1} \leq x \leq x_{k} \\
& =\frac{\left(x_{k+1}-x\right)^{2}}{\left(x_{k+1}-x_{k}\right)}-\frac{\left(x_{k+1}-x\right)^{3}}{\left(x_{k+1}-x_{k}\right)^{2}} \text { for } x_{k} \leq x \leq x_{k+1} \\
& =0 \text { elsewhere } \\
& H_{k}(y)=3\left(\frac{y-y_{k-1}}{y_{k}-y_{k-1}}\right)^{2}-2\left(\frac{y-y_{k-1}}{y_{k}-y_{k-1}}\right)^{3} \text { for } y_{k-1} \leq y \leq y_{k} \\
& =3\left(\frac{y_{k+1}-y}{y_{k+1}-y_{k}}\right)^{2}-2\left(\frac{y_{k+1}-y}{y_{k+1}-y_{k}}\right)^{3} \text { for } y_{k} \leq y \leq y_{k+1} \\
& =0 \text { elsewhere } \\
& S_{k}(y)=-\frac{\left(y-y_{k-1}\right)^{2}}{\left(y_{k}-y_{k-1}\right)}+\frac{\left(y-y_{k-1}\right)^{3}}{\left(y_{k}-y_{k-1}\right)^{2}} \text { for } y_{k-1} \leq y \leq y_{k} \\
& =\frac{\left(y_{k+1}-y\right)^{2}}{\left(y_{k+1}-y_{k}\right)}-\frac{\left(y_{k+1}-y\right)^{3}}{\left(y_{k+1}-y_{k}\right)^{2}} \text { for } y_{k} \leq y \leq y_{k+1} \\
& =0 \text { elsewhere }
\end{aligned}
$$

The approximate solution $\tilde{u}(x, y)$ has the form

$$
\tilde{u}(x, y)=\sum_{i=1}^{s_{x}} \sum_{j=1}^{s_{y}}\left\{A_{i j} H_{i}(x) H_{j}(y)+B_{i j} H_{i}(x) S_{j}(y)+C_{i j} S_{i}(x) H_{j}(y)+D_{i j} S_{i}(x) S_{j}(y)\right\}
$$


so the $N=4 s_{x} s_{y}$ unknowns are $A_{11}, B_{11}, C_{11}, D_{11}, A_{12}, B_{12}, C_{12}, D_{12}, \ldots, A_{s_{x} s_{y}}, B_{s_{x} s_{y}}$, $C_{s_{x} s_{y}}, D_{s_{x} s_{y}}$ which are relabeled to $a_{1}, \ldots, a_{N}$ for notational convenience. The approximating function $\tilde{u}(x, y)$ has a continuous mixed derivative $\tilde{u}_{x y}$ because the mixed derivative of each of the basis functions is continuous. To be succesful in this setting, the key feature of continuous mixed derivatives has to hold. This feature is not common to most FE methods in use today. The basis functions normally used by Galerkin methods do not even have continuous $\tilde{u}_{x}$ or $\tilde{u}_{y}$.

The approximate solution is required to satisfy the PDE exactly at the four collocation points, in each of the $\left(s_{x}-1\right)\left(s_{y}-1\right)$ subrectangles, and to satisfy the boundary conditions at certain points. The number of boundary collocation points plus the number of interior collocations points $4\left(s_{x}-1\right)\left(s_{y}-1\right)$, is equal to the number of basis functions $4 s_{x} s_{y}$, which is equal to the number of unknowns $N$. Finding the $N$ parameters $a_{1}, \ldots, a_{N}$ results in a system of non-linear equations:

$$
\begin{aligned}
f_{1}\left(a_{1}, \ldots, a_{N}\right) & =0 \\
& \vdots \\
f_{N}\left(a_{1}, \ldots, a_{N}\right) & =0
\end{aligned}
$$

This system of non-linear equations is solved via Newton's method. The integration of time is similar to the Galerkin finite element method. Spatial variables are discretized with finite elements while time is treated with finite differences. This can be visualized as the non-linear elliptic operator $L(u)$ evolving through time. Each equation corresponds to a collocation point. The dynamic counterpart to eq. (31) is given by

$$
\dot{\boldsymbol{a}}=\left(\begin{array}{c}
\dot{a}_{1} \\
\vdots \\
\dot{a}_{N}
\end{array}\right)=\left(\begin{array}{c}
g_{1}\left(a_{1}, \ldots, a_{N}\right) \\
\vdots \\
g_{N}\left(a_{1}, \ldots, a_{N}\right)
\end{array}\right)
$$

This stiff system of non-linear ordinary differential equations can be solved with various timestepping procedures. We have chosen a Crank-Nicolson implementation. The initial conditions to eq. (32) are given by a discretization of the final condition of the PDE. All computations have been performed with PDE2D, a general purpose finite element solver described in [14].

\subsection{Estimating the Data}

Normally, there are not enough options in the market to compute implied correlations from market data. When the option is exchange-traded, it is possible to construct a historical data series of implicit correlations. As a first approach, one can pick the extremes of this time series as $\rho_{\max }$ and $\rho_{\min }$. For OTC products, a time series of historical correlations as in fig. 1 gives some insights into the behavior of correlation. Another approach to find $\rho_{\min }$ and $\rho_{\max }$ is based on the forward Kolmogorov equation [11]. First, one has to specify the functions $a(\rho)$ and $b(\rho)$ in

$$
d \rho_{t}=a\left(\rho_{t}\right) d t+b\left(\rho_{t}\right) d X_{t}
$$

and estimate their parameters. The forward Kolmogorov equation with appropriate boundary and final conditions gives the probabilities that $\rho$ will breach a pre-specified band. The converse approach is also valid: The correlation distribution is determined at each time step. In case one does not want the correlation to breach the band with a $5 \%$ probability, one has to cut off the 0.025 quantiles at the left and right hand side of the distribution. These quantiles are used as lower and upper bounds. Usually, the lower and upper bounds are both functions of time. 


\section{Applications of the Model}

\subsection{Capped Calls on Baskets}

As a first example of a rainbow option, we consider a capped call on a basket of two equities. This is a common OTC product. Sometimes it is traded at exchanges as well. ${ }^{1}$ For pricing this call we have to solve PDE (13) - (15) with the following final and boundary conditions:

$$
\begin{aligned}
V(T) & =\min \left[\mathrm{cap}, \max \left(0, w_{1} S_{1}+w_{2} S_{2}-E\right)\right] \\
\Omega & =\left[S_{1}^{\text {min }} ; S_{1}^{\text {max }}\right] \times\left[S_{2}^{\text {min }} ; S_{2}^{\text {max }}\right] \\
V\left(S_{1}, S_{2}^{\text {min }}\right) & =B S\left(S_{1}, \frac{E}{w_{1}}, t\right)-B S\left(S_{1}, \text { cap }, t\right) \\
\frac{\partial V\left(S_{1}, S_{2}^{\text {max }}\right)}{\partial S_{2}} & =0 \\
V\left(S_{1}^{\text {min }}, S_{2}\right) & =B S\left(S_{2}, \frac{E}{w_{1}}, t\right)-B S\left(S_{2}, \text { cap }, t\right) \\
\frac{\partial V\left(S_{1}^{\text {max }}, S_{2}\right)}{\partial S_{1}} & =0
\end{aligned}
$$

The function $B S(x, y, t)$ denotes the price of a European call option with an underlying price of $x$ and a strike price of $y$ as given by the Black-Scholes model [1]. ${ }^{2}$

\begin{tabular}{|l|c|c|}
\hline Parameter & Symbol & Value \\
\hline \hline First asset price & $S_{1}$ & 100 \\
\hline Weight first asset & $w_{1}$ & 1 \\
\hline Second asset price & $S_{2}$ & 100 \\
\hline Weight second asset & $w_{2}$ & 1 \\
\hline Strike price & $E$ & 200 \\
\hline Interest rate & $r$ & 0.0953102 \\
\hline Dividend yield first asset & $D_{1}$ & 0.0487902 \\
\hline Dividend yield second asset & $D_{2}$ & 0.0 \\
\hline Time to Maturity & $\mathrm{T}$ & 0.5 \\
\hline \multirow{3}{*}{ Domain } & $S_{1}^{\text {min }}$ & 0 \\
\cline { 2 - 3 } & $S_{1}^{\text {max }}$ & 200 \\
\cline { 2 - 3 } & $S_{2}^{\text {min }}$ & 0 \\
\cline { 2 - 3 } & $S_{2}^{\text {max }}$ & 200 \\
\hline Cap & cap & 10 \\
\hline
\end{tabular}

Table 1: Data Capped Call on a Basket

\begin{tabular}{|c|c|c|c|c|c|}
\hline \multicolumn{2}{|c|}{ Volatility } & Scenario 1 & Scenario 2 & \multicolumn{2}{c|}{ Scenario 3} \\
\hline$\sigma_{1}$ & $\sigma_{2}$ & $\rho=0.5$ & $\rho^{-}=0.4 \leq \rho \leq 0.6=\rho^{+}$ & $\rho^{-}=0.3 \leq \rho \leq 0.7=\rho^{+}$ & $\delta_{\rho^{-}}$ \\
\hline & 0.1 & 5.3676 & 5.1953 & 5.0833 & -0.050 \\
\hline 0.1 & 0.2 & 4.9923 & 4.8832 & 4.7742 & -0.050 \\
\hline & 0.3 & 4.7555 & 4.6612 & 4.5677 & -0.045 \\
\hline & 0.1 & 4.9625 & 4.8878 & 4.7767 & -0.050 \\
\hline 0.2 & 0.2 & 4.9989 & 4.7348 & 4.5998 & -0.065 \\
\hline & 0.3 & 4.7120 & 4.5884 & 4.4569 & -0.065 \\
\hline & 0.1 & 4.7632 & 4.6669 & 4.5706 & -0.050 \\
\hline 0.3 & 0.2 & 4.7246 & 4.5686 & 4.4584 & -0.070 \\
\hline & 0.3 & 4.6329 & 4.5006 & 4.3591 & -0.070 \\
\hline
\end{tabular}

Table 2: Results Capped Call on a Basket

\footnotetext{
${ }^{1}$ In Germany, examples for capped options are WKN 822361, WKN 822362, WKN 822380, and WKN 822399 which are traded in Frankfurt, Düsseldorf, Stuttgart.

${ }^{2}$ For a discussion of the boundary conditions and alternative specifications see [16].
} 


\subsection{Options on the Maximum or the Minimum of Two Risky Assets}

This product has the payoff

$$
\left[\phi\left(\eta \min \left(\eta S_{1}, \eta S_{2}\right)-K\right)\right]^{+}
$$

This is a call $(\phi=+1)$ or put $(\phi=-1)$ on the maximum $(\eta=-1)$ or minimum $(\eta=+1)$ of the two underlyings $S_{1}$ and $S_{2}$ with a strike $K$. It has been shown by ([13], eq. (123)) that $\Gamma_{\text {cross }}$ can be expressed as:

$$
\begin{aligned}
\Gamma_{\text {cross }} & =\phi \eta \underbrace{\frac{e^{\left(r-D_{1}\right)(T-t)}}{S_{2} \sigma \sqrt{T-t}}}_{=B} n\left(d_{3}\right) N\left(\phi \sigma \frac{d_{3} \rho_{1}-d_{1}}{\sigma_{2} \sqrt{1-\rho^{2}}}\right) \\
\rho_{1} & =\frac{\rho \sigma_{2}-\sigma_{1}}{\sigma} \\
\sigma^{2} & =\sigma_{1}^{2}+\sigma_{2}^{2}-2 \rho \sigma_{1} \sigma_{2} \\
d_{1} & =\frac{\ln \left(\frac{S_{1}}{E}\right)+\left(r-D_{1}+\frac{1}{2} \sigma_{1}^{2}\right)(T-t)}{\sigma_{1} \sqrt{T-t}} \\
d_{3} & =\frac{\ln \left(\frac{S_{2}}{S_{1}}\right)+\left(D_{1}-D_{2}-\frac{1}{2} \sigma^{2}\right)(T-t)}{\sigma \sqrt{T-t}}
\end{aligned}
$$

The functions $N(\cdot)$ and $n(\cdot)$ represent the cumulative distribution function and the density function of the standard normal distribution, respectively. Since $B, N(\cdot)$ and $n(\cdot)$ are positive, the sign of $\Gamma_{\text {cross }}$ depends solely on the variables $\phi$ and $\eta$ which determine the product type.

\begin{tabular}{|c|c|c|c|}
\hline Product & $\phi$ & $\eta$ & $\Gamma_{\text {cross }}$ \\
\hline \hline Call on the Maximum & +1 & -1 & ${ }^{\prime}-^{\prime}$ \\
\hline Call on the Minimum & +1 & +1 & ${ }^{\prime}+^{\prime}$ \\
\hline Put on the Minimum & -1 & +1 & ${ }^{\prime}-^{\prime}$ \\
\hline Put on the Maximum & -1 & -1 & ${ }^{\prime}+^{\prime}$ \\
\hline
\end{tabular}

Table 3: Signs of $\Gamma_{\text {cross }}$

Using these results, the worst-case scenarios can be computed using the Black-Scholes price with the following correlations [2]:

\begin{tabular}{|c|l|}
\hline Product & \multicolumn{1}{|c|}{ Correlation } \\
\hline \hline Call on the Maximum & $\rho_{\text {Average }}^{+}=\frac{1}{T-t} \int_{t}^{T} \rho^{+}(\tau) d \tau$ \\
\hline Call on the Minimum & $\rho_{\text {Average }}^{+}=\frac{1}{T-t} \int_{t}^{T} \rho^{+}(\tau) d \tau$ \\
\hline Put on the Minimum & $\rho_{\text {Average }}^{-}=\frac{1}{T-t} \int_{t}^{T} \rho^{-}(\tau) d \tau$ \\
\hline Put on the Maximum & $\rho_{\text {Average }}^{-}=\frac{1}{T-t} \int_{t}^{T} \rho^{-}(\tau) d \tau$ \\
\hline
\end{tabular}

Table 4: Worst-Case Correlations

In the case when $\rho^{+}(t)$ is a constant, $\rho_{\text {Average }}^{+}$reduces to $\rho^{+}$. This holds in a similar fashion for $\rho^{-}(t)$.

\subsection{Two-Asset Barrier Options}

In October 1993, Bankers Trust structured a call option on a basket of Belgian stocks that would knock out if the Belgian franc -not the Belgian stock- appreciated by more than $3.5 \%$. So we have two spatial variables entering the pricing equation: one that determines the payoff treating the basket as a single risk factor, and one that determines whether the option is still alive. This kind of product went through a boom in the OTC market in 1997 with a strike in the exchange rate of USD vs. DEM and a barrier in the exchange rate in USD vs. FRF. Back then, this product was employed to profit from the high difference in historical and implied correlation. Normally, this product comes with an FX rate on the knock-out barrier so that 
an adjustment for discrete monitoring of the barrier is not necessary. This product can be generalized for knock-in and part-time barriers so that the barrier is only valid for parts of the life of the option [5].

We again solve the PDE eq. (13) - (15) with the following final and boundary conditions:

$$
\begin{aligned}
V(T) & =\max \left(0, w_{2} S_{2}-E\right) \\
\Omega & =\left[S_{1}^{\text {min }} ; S_{1}^{\text {max }}\right] \times\left[S_{2}^{\text {min }} ; S_{2}^{\text {max }}\right] \\
\frac{\partial V\left(S_{1}, S_{2}^{\text {min }}\right)}{\partial S^{2}} & =0 \\
\frac{\partial V\left(S_{1}, S_{2}^{\text {max }}\right)}{\partial S_{2}} & =1 \\
V\left(S_{1}^{\text {min }}, S_{2}\right) & =0 \\
\frac{\partial V\left(S_{1}^{\text {max }}, S_{2}\right)}{\partial S_{1}} & =0
\end{aligned}
$$

and the data:

\begin{tabular}{|l|c|c|}
\hline Parameter & Symbol & Value \\
\hline \hline First asset price & $S_{1}$ & 100 \\
\hline Weight first asset & $w_{1}$ & 1 \\
\hline Weight second asset & $w_{2}$ & 1 \\
\hline Strike price & $E$ & 100 \\
\hline Interest rate & $r$ & 0.1 \\
\hline Dividend yield first asset & $D_{1}$ & 0.0 \\
\hline Dividend yield second asset & $D_{2}$ & 0.0 \\
\hline Volatility first asset & $\sigma_{1}$ & 0.2 \\
\hline Volatility second asset & $\sigma_{2}$ & 0.3 \\
\hline Time to Maturity & $\mathrm{T}$ & 0.5 \\
\hline Correlation & $\rho$ & varies \\
\hline \multirow{3}{*}{ Domain } & $S_{1}^{\text {min }}$ & varies \\
\cline { 2 - 3 } & $S_{1}^{\text {max }}$ & 300 \\
\cline { 2 - 3 } & $S_{2}^{\text {min }}$ & 0 \\
\cline { 2 - 3 } & $S_{2}^{\text {max }}$ & 300 \\
\hline
\end{tabular}

Table 5: Data Two-Asset Barrier Option Product

\begin{tabular}{|c|c|c|c|c|c|c|}
\hline \multirow{3}{*}{$S_{2}$} & \multicolumn{6}{|c|}{ Location of Barrier } \\
\cline { 2 - 7 } & \multicolumn{3}{|c}{95} & \multicolumn{3}{|c|}{99} \\
\cline { 2 - 7 } & \multicolumn{2}{|c|}{0.5} & $0.4 \leq \rho \leq 0.6$ & \multicolumn{2}{|c|}{$\rho=0.5$} & $0.4 \leq \rho \leq 0.6$ \\
\cline { 2 - 7 } & Anal. & FE & FE & Anal. & FE & FE \\
\hline \hline 80 & 1.3373 & 1.3353 & 1.2257 & 0.3570 & 0.3568 & 0.3143 \\
\hline 85 & 2.1369 & 2.1320 & 1.9643 & 0.5572 & 0.5560 & 0.4945 \\
\hline 90 & 3.1633 & 3.1566 & 2.9205 & 0.8080 & 0.8064 & 0.7233 \\
\hline 95 & 4.4008 & 4.3939 & 4.0836 & 1.1039 & 1.1026 & 0.9969 \\
\hline 100 & 5.8210 & 5.8155 & 5.4230 & 1.4376 & 1.4368 & 1.3090 \\
\hline 105 & 7.3901 & 7.3874 & 6.9287 & 1.8007 & 1.8008 & 1.6524 \\
\hline 110 & 9.0741 & 9.0753 & 8.5487 & 2.1859 & 2.1870 & 2.0199 \\
\hline 115 & 10.8430 & 10.8485 & 10.2603 & 2.5868 & 2.5890 & 2.4051 \\
\hline 120 & 12.6721 & 12.6821 & 12.9316 & 2.9984 & 3.0017 & 2.8030 \\
\hline
\end{tabular}

Table 6: Results European Two-Asset Barrier Option

\subsection{Basket Options}

It holds for both calls and puts that the higher the correlation, the higher the price of the option. Consequently

$$
\frac{\partial V}{\partial \rho}=\kappa \geq 0
$$


From the following relationship ([13], eq. (100))

$$
\kappa=\underbrace{\sigma_{1} \sigma_{2} \tau S_{1} S_{2}}_{\geq 0} \Gamma_{12}
$$

we can conclude that $\Gamma_{12}$ is always positive. Therefore, the worst-case price of a basket option only depends on $\rho^{-}$. Since no closed-form solution is known for basket options, the price of this type of option has to be computed numerically or by using an analytical approximation. For a survey of available methods see [6].

\section{Conclusions and Outlook}

For many rainbow options, the worst case scenarios cannot be computed by simply plugging in the worst case correlation into the Black-Scholes models. Whenever numerical techniques need to be used, they need to provide a good approximation of the mixed derivative. One method that does well under these circumstances is a collocation finite element method with a cubic Hermite basis function.

Other common OTC products are rainbows with a binary payoff structure and various caps or barriers. Digital payoffs lead to high curvature in the spatial variables, a feature finite elements can easily deal with. Barriers introduce non-rectangular domains (compare [16]) which are hard to cover with finite differences while they pose no problem for finite element methods. Recently, some American rainbows have been sold. ${ }^{3}$ Currently, no models are available that describe the worst-case price in the presence of an early exercise. Also, since most basket options have more than just two underlyings, solvers in higher dimensions need to be developed.

\footnotetext{
${ }^{3}$ For example: Deutsche Bank has issued various American options on baskets of Hewlett Packard Co./Agilent Technology (WKN 845950ff) and Roche Holding AG/Givaudan AG (WKN 836746ff) which are listed at various German stock exchanges.
} 


\section{References}

[1] Black, F., Scholes, M.: The Pricing of Options and Corporate Liabilities. Journal of Political Economy, 81 (1973) 637-659.

[2] Bos, L. P., Ware, A. F.: How to Solve Multiasset Black-Scholes with Time-dependent Volatility and Correation. Journal of Computational Finance. 4/2 (2000) 99-107.

[3] Forsyth, P. A., Vetzal, K. R., Zvan, R.: A General Finite Element Approach for PDE Option Pricing Models. Preprint University of Waterloo. Version December 1998.

[4] Forsyth, P. A., Vetzal, K. R., Zvan, R.: An Unstructured Meshing for Two Asset Barrier Options. Applied Mathematical Finance. 7 (33-60) 2000.. Version October 1999.

[5] Haug, E. G.: The Complete Guide to Option Pricing Formulas. New York, 1997.

[6] Heurich, M., Topper, J.: Pricing Basket Options - A Comparative Study. Discussion Paper University of Hannover No. 223. [In Preparation]

[7] Hunziker, J. P., Koch-Medina P.: Two-Color Rainbow Options in: [10].

[8] Jackson, N., Süli, E.: Adaptive Finite Element Solution of $1 D$ European Option Pricing Problems. Oxford University Computing Laboratory. Report 97/05.

[9] Lopez, J. A., Walter, Chr. A.: Is Implied Correlation Worth Calculating? Evidence from Foreign Exchange Options. Journal of Derivatives. 7 (Spring 2000) 65-81.

[10] Nelken, Israel: The Handbook of Exotic Options. Chicago etc., 1996.

[11] Oztukel, A., Wilmott, P.: Uncertain Parameters, an Empirical Stochastic Volatility Model and Confidence Limits. Preprint Oxford University, March 1996.

[12] Radde, K.-U.: Reverse Dual Currency Bond (Triple Currency Linked Bond) in: Eller, R., Gruber, W., Reif, M. (eds.): Handbuch Strukturierter Kapitalmarktprodukte. Stuttgart, 1999.

[13] Reiß, O., Wystup, U.: Efficient Computation of Option Price Sensitivities Using Homogeneity and other Tricks. Preprint Weierstraß-Institut for Applied Analysis und Stochastics. Berlin, Version 24.5.2000.

[14] Sewell, G.: Finite Differences, Finite Elements and PDE2D. El Paso, 2000. (http://members.aol.com/pde2d)

[15] Tavella, D.: Alternating Directions II. Risk. 14 (4/2001) 8.

[16] Topper, J.: Finite Element Modeling of Exotic Options. Discussion Paper No. 216, University of Hannover, Version Dec. 27, 1998.

[17] Topper, J.: Reverse Convertibles and Uncertain Parameters. Risk. 14 (2001).

[18] Yanenko, N. N. The Method of Fractional Steps. New York etc., 1971.

[19] Wilmott, P.: Derivatives - The Theory and Practice of Financial Engineering. Chichester etc., 1998. 